\title{
Cryptococcal meningitis initially presenting with eye symptoms in an immunocompetent patient: A case report
}

\author{
JUN LI $^{1}$, PEIPEI WANG ${ }^{2}$, LING YE $^{1}$, YANFANG WANG ${ }^{1}$, XIUZHEN ZHANG $^{1}$ and SONGPING YU ${ }^{1}$ \\ Departments of ${ }^{1}$ Ophthalmology and ${ }^{2}$ Stomatology, Lishui Hospital Affiliated to Zhejiang University, \\ Lishui, Zhejiang 323000, P.R. China
}

Received April 5, 2015; Accepted May 5, 2016

DOI: $10.3892 /$ etm. 2016.3440

\begin{abstract}
Although cryptococcal meningitis (CM) typically occurs in immunocompromised hosts, immunocompetent humans are susceptible to CM. In humans with an intact immune system, CM presents with signs and symptoms typical of meningitis, including fever, headache and neck stiffness. The present study reported the case of a female immunocompetent patient who presented visual blurring in both eyes and bilateral papilledema for $\sim 1$ month. Following hospital admission, the patient was diagnosed with optic nerve inflammation and was treated with intravenous methylprednisolone and oral prednisone. However, the initial symptoms failed to improve and the patient developed a headache. The microscopic examination and India ink test performed using the cerebrospinal fluid of the patient showed the presence of Cryptococcus neoformans. Following combined treatment with amphotericin B and fluconazole, the patient made a full recovery with total resolution of the initial symptoms. This case demonstrates that $\mathrm{CM}$ in immunocompetent patients may initially include eye symptoms, which may result in a delayed diagnosis of CM.
\end{abstract}

\section{Introduction}

Cryptococcal meningitis (CM) is a burden disease in middleand low-income countries with over 1 million cases and 600,000 mortalities per year (1). CM caused by the fungus Cryptococcus neoformans remains a leading cause of morbidity and mortality among immunocompromised patients, such as those with a malignancy, connective tissue disorder, immunodeficiency virus (HIV) infection or having undergone organ transplantation (2). However, immunocompetent patients are susceptible to $\mathrm{CM}$ and can present in the clinic with signs and symptoms typical of meningitis, such as fever, headache and

Correspondence to: Professor Songping Yu, Department of Ophthalmology, Lishui Hospital Affiliated to Zhejiang University, 289 Kuocang Road, Lishui, Zhejiang 323000, P.R. China

E-mail: yusongpin@126.com

Key words: immunocompetent, cerebrospinal fluid, cryptococcal meningitis, papilledema neck stiffness (3). Treatment of CM consists of three phases: Induction, consolidation, and maintenance; effective induction therapy requires potent fungicidal drugs $(2,4)$.

Loss of vision, diplopia and ocular motility deficit have been reported previously (5-7). In the present study, a female immunocompetent patient with CM is reported who initially presented with eye symptoms, and subsequently fully recovered with complete resolution of the initial symptoms following combined treatment with amphotericin B and fluconazole.

\section{Case report}

A 42-year-old female presented in September 2014 at the Department of Ophthalmology (Lishui Hospital Affiliated to Zhejiang University, Lishui, China) with complaints of blurred vision in both eyes and intermittent fatigue for $\sim 1$ month. No additional symptoms, such as ocular pain, diplopia, headache, nausea or vomiting, were reported. The patient had no previous history of medical illness. The patient was admitted to the hospital for further evaluation. The study was approved by the Human Research Ethics Committee of Lishui Hospital Affiliated to Zhejiang University, and was performed according to the Declaration of Helsinki. Written informed consent was obtained from the patient for publication of this Case report and the accompanying images.

A general examination performed upon hospital admission demonstrated that the clinical parameters, including height, body weight and body mass index, of the patient were within normal limits. The patient was afebrile and her vital signs were normal. An ocular examination indicated uncorrected distance visual acuities of 1.0 and 0.8 in the right and left eye, respectively. An automated visual field test using the 30-2 fast threshold program included with the Humphrey Visual Field Analyzer (Humphrey 750i; Carl Zeiss AG, Oberkochen, Germany) showed marginally enlarged blind spots in both eyes (Fig. 1), and a slit lamp examination showed quiet anterior chambers without significant abnormalities or an afferent pupillary defect. Fundoscopy revealed marked elevated discs and evidence of bilateral splinter optic disc hemorrhaging (Fig. 2). The maculae, retinal vasculature and retinal peripheries were normal. Fluorescein (Alcon, Shanghai, China) angiography showed staining of the optic disc and late peripapillary dye pooling (Fig. 3). The intraocular pressure of the patient remained in the normal range 


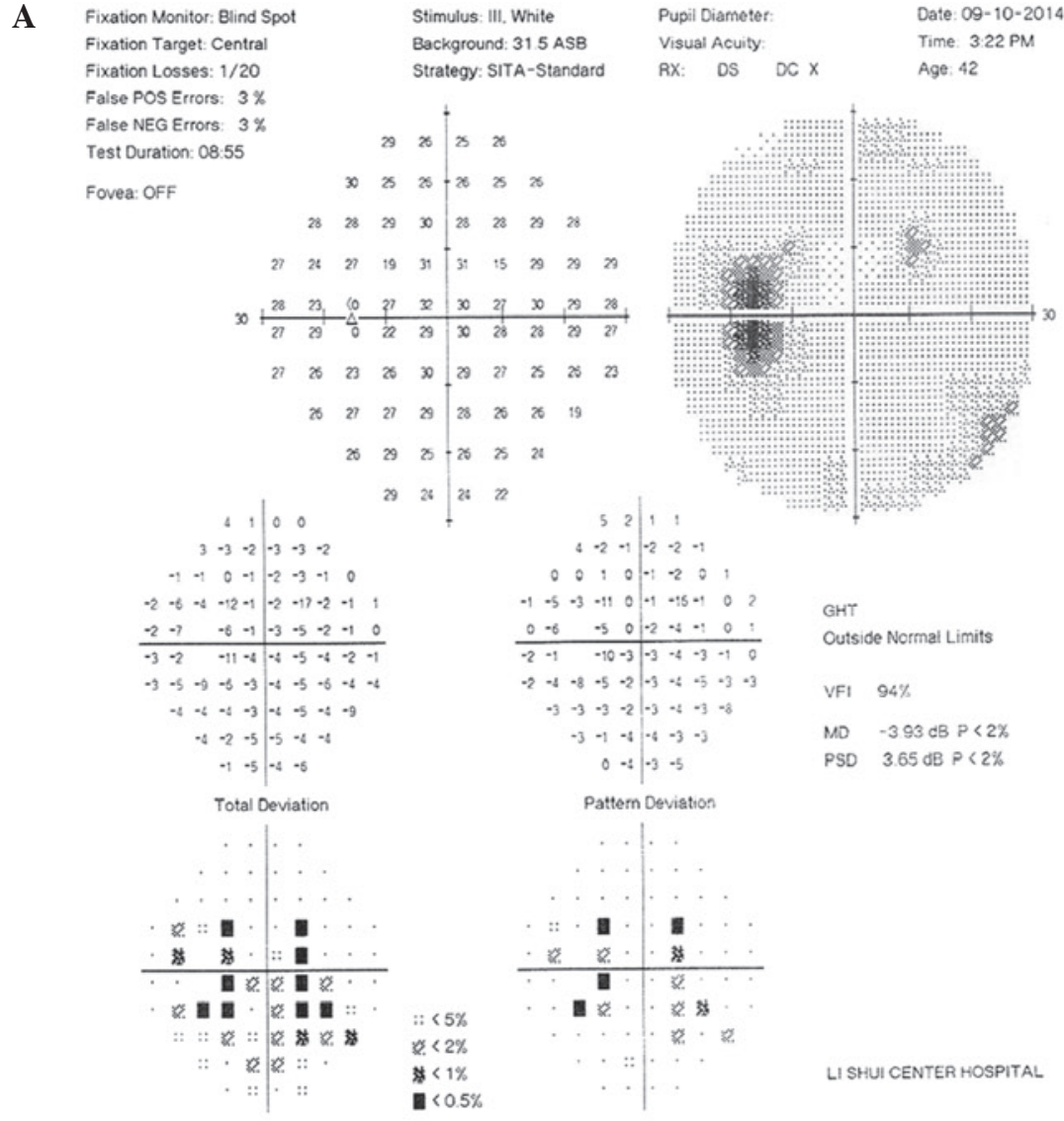

B

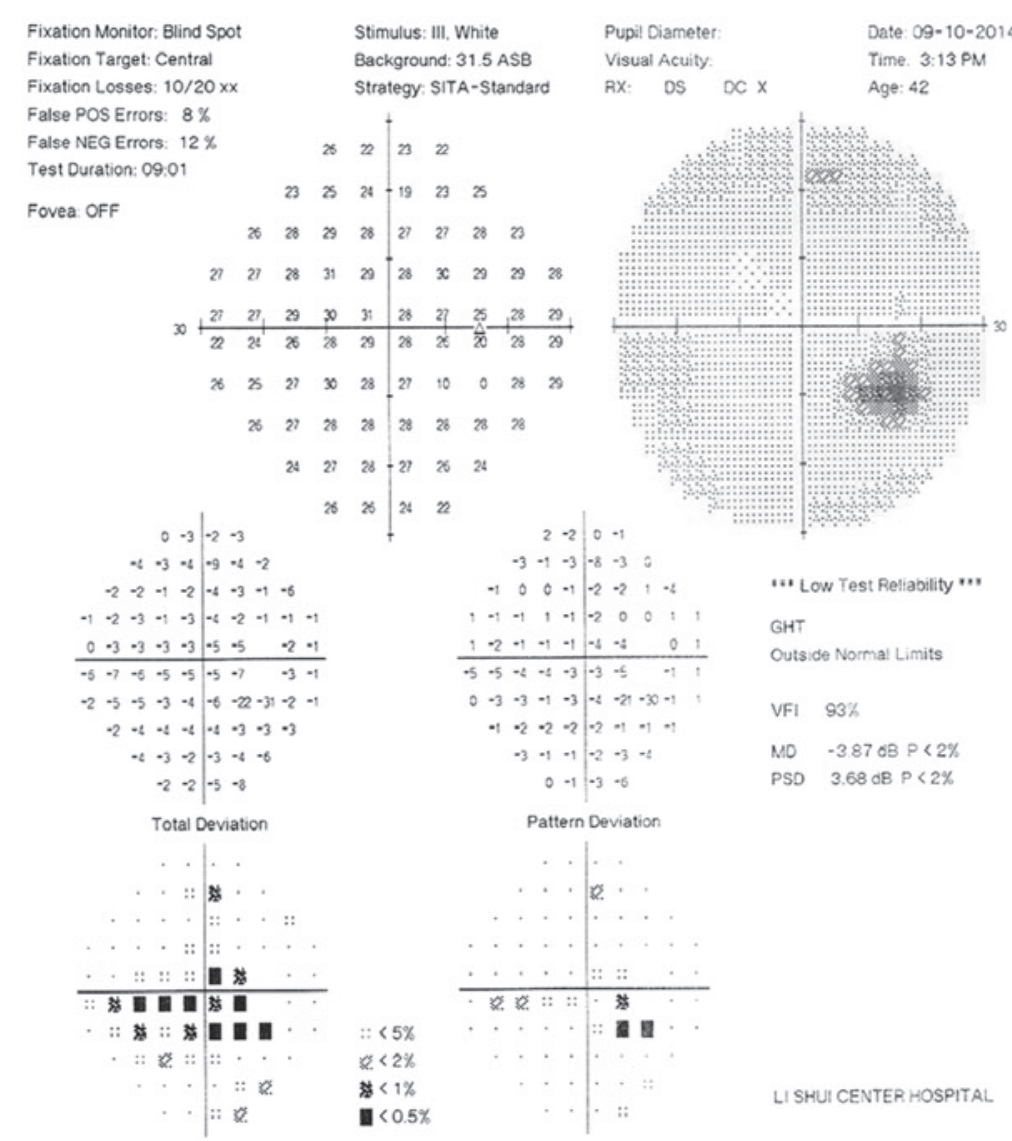

Figure 1. The 30-2 program of the Humphrey Visual Field Analyzer indicating slightly enlarged blind spots in the (A) left and (B) right eyes. RX, prescription of ametropia; DS, spherical power; DC, astigmatic power; MD, mean deviation; PSD, pattern standard deviation; VFI, visual field index; GHT, glaucoma hemifield test. 

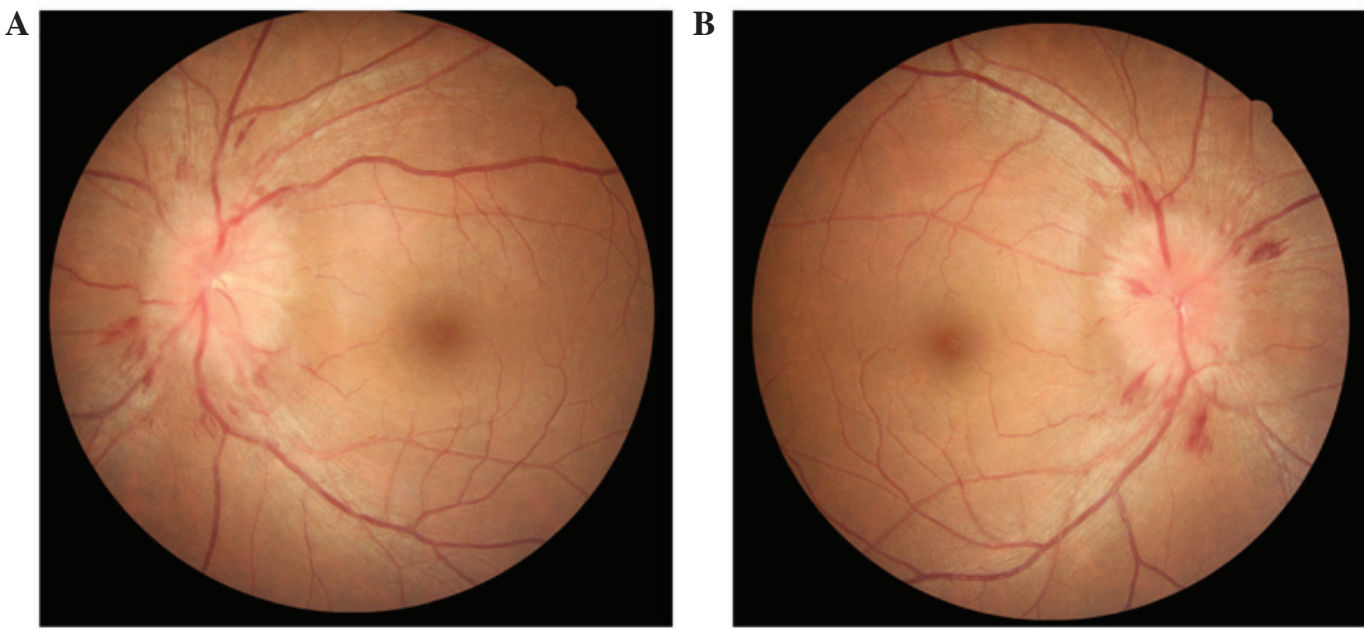

Figure 2. Fundus photographs showing (A) left and (B) right eyes with established papilledema (hyperemia, blurring of the optic disc margins, venous congestion of peripapillary vessels and peripapillary splinter hemorrhages).
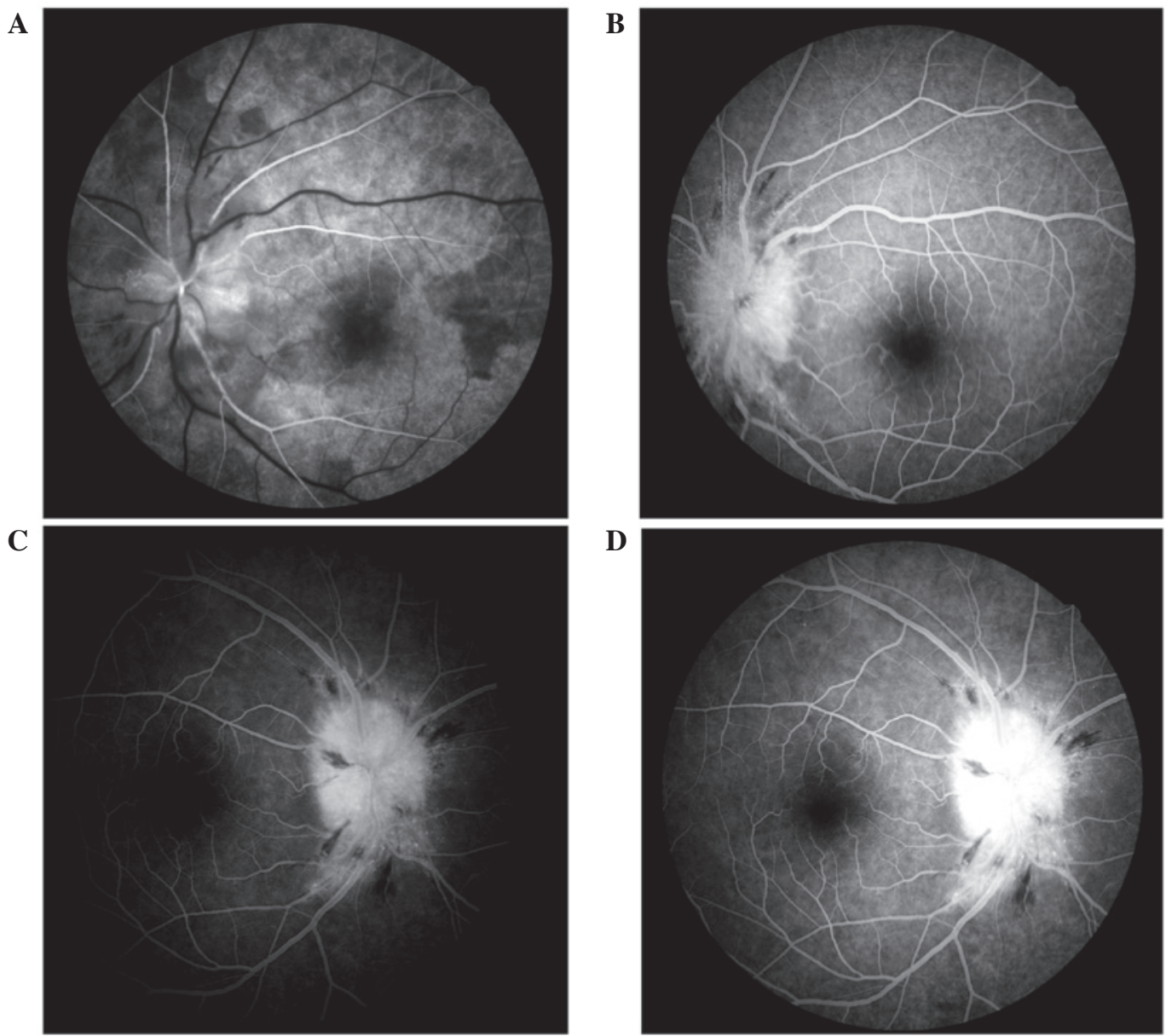

D

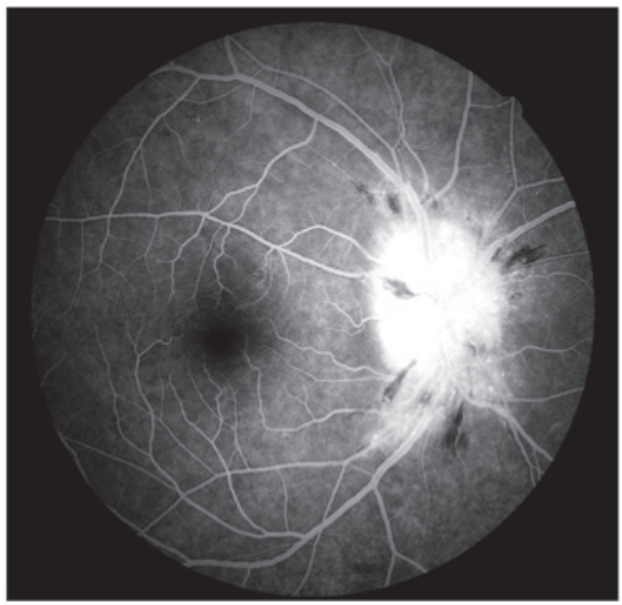

Figure 3. Fundus fluorescein angiography photographs of left (upper) and right (lower) eyes showing staining of the optic disc in the (A and C) early phases of cryptococcal meningitis infection. (B and D) Peripapillary dye pooling in the late phases of infection.

(10-21 $\mathrm{mmHg}$ ) throughout all examinations, and there was no ptosis, proptosis or chemosis. A motor and sensory examination of both eyes showed normal results, and the remainder of the neurological examination indicated the following characteristics: Clear and co-operative mentality, with normal advanced neural activity; no atrophy in the limb muscles; normal muscle tension; muscle strength grade $\mathrm{V}$; positive tendon reflex of the limbs; negative bilateral Hoffmann reflex, with the bilateral Babinski reflex not being elicited; normal gait; and no ataxia. A chest X-ray (Fig. 4) and brain magnetic resonance imaging (MRI) scan (Fig. 5) showed no evident abnormalities. 


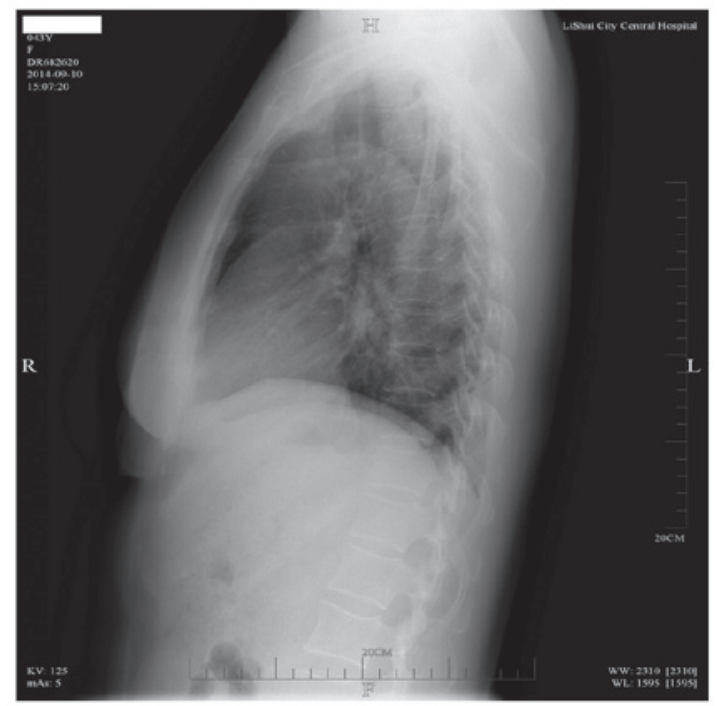

Figure 4. Chest X-ray revealing minimal interstitial fibrosis in the upper left lung.

The patient's initial diagnosis following admission was optic nerve inflammation, and the patient was treated with intravenous (IV) methylprednisolone (1 g/day for 3 days; Pfizer, Inc., New York, NY, USA) followed by oral prednisone (1 mg/day for 11 days; Xianju Pharmaceutical Co., Itd., Taizhou, China). On day 18 following admission, the patient reported having a persistent stabbing headache, but did not display other symptoms, such as nausea, vomiting, fever, neck stiffness or confusion. It was considered that the papilledema of the patient may be secondary to a prolonged increase in intracranial pressure and infectious infiltration of the optic nerve sheath. A lumbar puncture demonstrated an opening pressure $>400 \mathrm{mmH}_{2} \mathrm{O}$, and an analysis of cerebrospinal fluid (CSF), including cell counts, measurements of protein and glucose levels, showed a markedly elevated protein concentration $(0.77 \mathrm{~g} / \mathrm{l}$; reference range, 0.2-0.4 g/l) and decreased glucose concentration (1.08 $\mathrm{mmol} / \mathrm{l}$; reference range, $2.5-0.4 \mathrm{mmol} / \mathrm{l})$. The results of a common bacterial culture, smear and culture for acid fast bacilli in the CSF were negative; however, a microscopic examination (CX31; Olympus Corporation, Tokyo, Japan) and India ink test (Yuanmu Biological Technology Co., Ltd., Shanghai, China), performed according to the manufacturer's instructions as described in a previous study (8), showed the presence of Cryptococcus neoformans. The results of other laboratory tests, including an enzyme-linked immunosorbent assay for toxoplasmosis (cat. no. S20020001; Haitai Biological Pharmaceutical Co., Ltd., Zhuanghe, China), treponema pallidum hemagglutination (cat. no. NV30918; Jianlun Science and Technology Co., Ltd., Guangzhou, China) tests and an HIV antibody test (cat. no. S19990046; Zhongshan Biological Pharmaceutical Co., Ltd., Zhongshan, China) were all negative.

Based on the clinical symptoms and positive microscopic examination and India ink test results, the patient was diagnosed with CM. Following diagnosis, the patient was treated with amphotericin B (IV; $1.0 \mathrm{mg} / \mathrm{kg} / \mathrm{day}$; North China Pharmaceutical Co., Ltd., Shijiazhuang, China) in combination with oral fluconazole (200 mg, twice daily; Bokang Gene Science and Technology Co., Ltd., Beijing, China). Additionally,
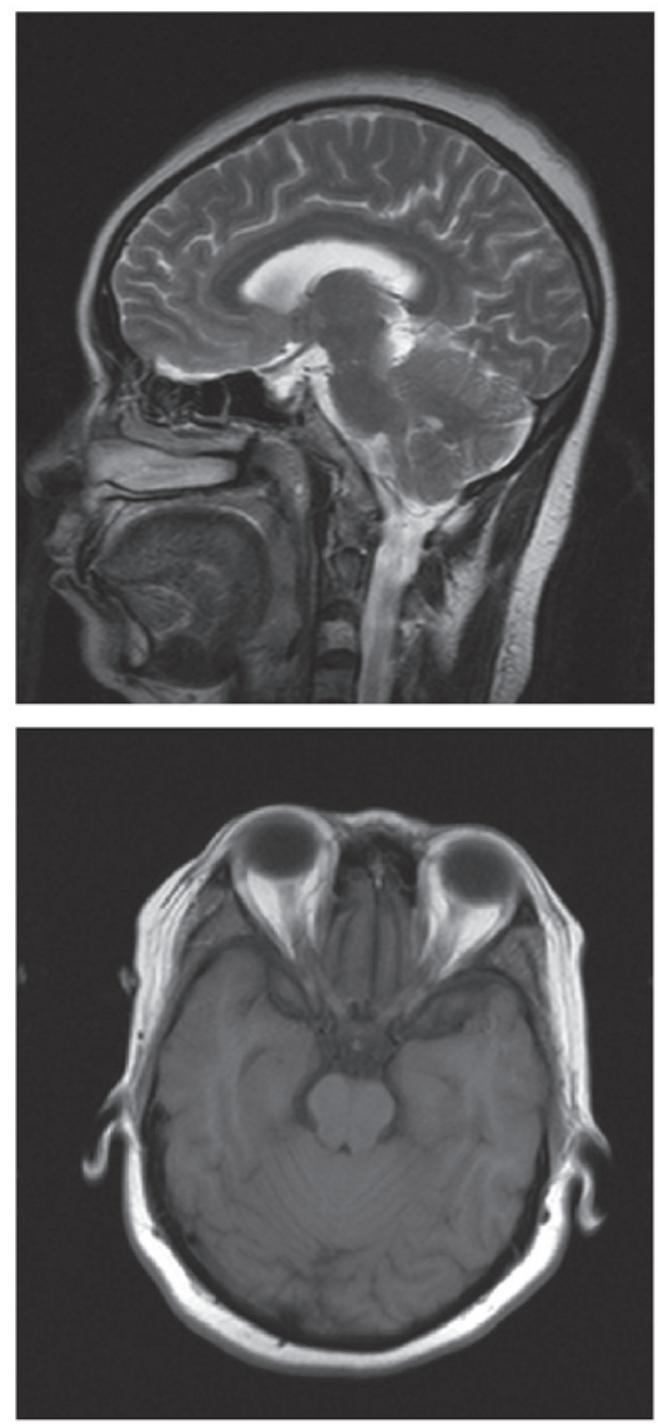

Figure 5. Magnetic resonance imaging of the brain showing normal findings.

IV mannitol (125 ml, twice daily; Shuanghe Pharmaceutical Co., Ltd, Wuhan, China) and oral acetazolamide (200 mg, twice daily; Aoyibaoling Pharmaceutical Co., Ltd., Hangzhou, China) were administered during the early phase of drug therapy to control the elevated intracranial pressure. Following treatment for 1.5 months, the patient fully recovered her visual acuity and regained a normal optic disc. At this point, previous medications were halted and the patient began a 2-month course of mono-treatment with oral fluconazole (400 mg/day). After 2 months of treatment with oral fluconazole, the results of a repeat CSF examination were negative for Cryptococcus neoformans, and the patient showed no evidence of disease recurrence at a 3 month follow-up visit.

\section{Discussion}

Cryptococcosis, caused by Cryptococcus neoformans, is an important infectious disease with a worldwide presence $(1,9,10)$. Cryptococci are encapsulated saprophytic yeasts often found in soil contaminated with avian excreta (11). Humans typically become infected by inhaling the aerosolized organisms, following which the infection can be asymptomatic or limited 
to the lungs $(11,12)$; however, the infection can later spread hematogenously to other areas of the body, in particular to the meninges (3).

Compared with its presence in immunocompetent patients, Cryptococcosis is more common in immunocompromised patients, including those with impaired cell-mediated immunity (13). Such impaired patients include those with a malignancy, connective tissue disorder, HIV infection or those who have undergone organ transplantation $(2,14)$. However, previous studies have shown that a number of patients infected with Cryptococcosis are immunocompetent $(6,15-17)$. CM causes morbidity and mortality worldwide, particularly in patients with impaired cell-mediated immunity; however, the clinical outcomes in immunocompetent patients are more favorable.

The initial clinical manifestations of a $\mathrm{CM}$ infection are highly variable. In non-immunocompromised patients, CM typically presents with signs and symptoms associated with meningitis, such as a fever, headache and neck stiffness $(3,18)$. Additionally, cranial neuropathies and ophthalmoplegia are common complications in patients with CM $(5,6,16,19)$. However, primary bilateral blurred vision without any accompanying symptoms is a rare initial presentation of a cryptococcal infection. In the current case, the only abnormalities of the patient upon presentation consisted of decreased uncorrected distance visual acuity in both eyes and bilateral papilledema, which was present for $\sim 1$ month. Other common signs and symptoms that could be attributed to intracranial pressure, such as headache, confusion, nausea and vomiting, were absent upon admission. Such a presentation may result in the delayed diagnosis of a brain disease.

A satisfactory evaluation for CM requires a lumbar puncture with measurement of the opening pressure, and subsequent CSF analysis, including measurements of protein and glucose expression levels. In addition, India ink staining, a fungal culture and cryptococcal antigen tests should be performed (20). A definitive diagnosis of CM requires visualizing the fungus in CSF using the India ink test, positive CSF latex agglutination assay results or CSF culture results positive for Cryptococcus neoformans (21). In the present case, $\mathrm{CM}$ was diagnosed based on results of the India ink test, which, according to previous reports, has a sensitivity of $75-85 \%$. Additionally, increased protein expression levels and decreased glucose expression levels in CSF may indicate the presence of a more severe inflammation, which is concordant with the results in the present study. Imaging tests may also provide information useful for diagnosing CM. For example, a communicating hydrocephalus may occur early in a $\mathrm{CM}$ infection due to acute meningeal exudate, and can also occur in a late-stage infection due to the presence of meningeal adhesions (22). However, in the present case, the cranial MRI scan of the patient upon hospital admission was normal, and showed no evidence of cranial infection or increased intracranial pressure.

The optimal treatment regimen and duration for a Cryptococcosis infection in non-HIV-infected patients remains largely unknown (23). Current recommendations are derived from previous clinical experience with HIV-infected individuals, but are not supported with evidence obtained from large randomized controlled clinical trials. In the current study, the patient was treated with amphotericin B (IV; $1.0 \mathrm{mg} / \mathrm{kg} /$ day) administered in conjunction with fluconazole (oral; $400 \mathrm{mg} /$ day). It has been reported that using a dose of amphotericin B (0.7-1 mg/kg/day) may result in a slightly lower mortality rate (24).

In conclusion, the present study demonstrates that eye symptoms may constitute the initial presentation of CM in an immunocompetent patient, and such symptoms can lead to its delayed diagnosis. CM should be suspected in any cases of decreased visual acuity of unknown origin accompanied with unexplained bilateral papilledema in patients with or without a compromised immune system.

\section{References}

1. Park BJ, Wannemuehler KA, Marston BJ, Govender N, Pappas PG and Chiller TM: Estimation of the current global burden of cryptococcal meningitis among persons living with HIV/AIDS. AIDS 23: 525-530, 2009.

2. Sloan DJ and Parris V: Cryptococcal meningitis: Epidemiology and therapeutic options. Clin Epidemiol 6: 169-182, 2014.

3. Chen SC, Slavin MA, Heath CH, Playford EG, Byth K, Marriott D, Kidd SE, Bak N, Currie B, Hajkowicz K, et al; Australia and New Zealand Mycoses Interest Group (ANZMIG)-Cryptococcus Study: Clinical manifestations of Cryptococcus gattii infection: Determinants of neurological sequelae and death. Clin Infect Dis 55: 789-798, 2012.

4. Pappalardo MC, Szeszs MW, Martins MA, Baceti LB, Bonfietti LX, Purisco SU, Baez AA and Melhem MS: Susceptibility of clinical isolates of Cryptococcus neoformans to amphotericin B using time-kill methodology. Diagn Microbiol Infect Dis 64: 146-151, 2009.

5. Muslikhan Y, Hitam WH, Ishak SR, Mohtar I and Takaran J: Cryptococcus meningitis in an immunocompetent teenage boy presented early with diplopia. Int J Ophthalmol 3: 92-94, 2010.

6. Liyanage DS, Pathberiya LP, Gooneratne IK, Caldera MH, Perera PW and Gamage R: Cryptococcal meningitis presenting with bilateral complete ophthalmoplegia: A case report. BMC Res Notes 7: 328, 2014.

7. Moodley A, Rae W, Bhigjee A, Connolly C, Devparsad N, Michowicz A, Harrison T and Loyse A: Early clinical and subclinical visual evoked potential and Humphrey's visual field defects in cryptococcal meningitis. PloS One 7: e52895, 2012.

8. Saha DC, Xess I and Jain N: Evaluation of conventional \& serological methods for rapid diagnosis of cryptococcosis. Indian J Med Res 127: 483-488, 2008.

9. Leal AL, Faganello J, Fuentefria AM, Boldo JT, Bassanesi MC and Vainstein MH: Epidemiological profile of cryptococcal meningitis patients in Rio Grande do Sul, Brazil. Mycopathologia 166: 71-75, 2008.

10. Pyrgos V, Seitz AE, Steiner CA, Prevots DR and Williamson PR: Epidemiology of cryptococcal meningitis in the US: 1997-2009. PloS One 8: e56269, 2013.

11. Perfect JR and Casadevall A: Cryptococcosis. Infecti Dis Clin North Am 16: 837-874, 2002.

12. Corti M, Solari R, Cangelosi D, Domínguez C, Yampolsky C, Negroni R, Arechavala A and Schtirbu R: Sudden blindness due to bilateral optic neuropathy associated with cryptococcal meningitis in an AIDS patient. Rev Iberoam Micol 27: 207-209, 2010.

13. Schimpff SC and Bennett JE: Abnormalities in cell-mediated immunity in patients with Cryptococcus neoformans infection. J Allergy Clin Immunol 55: 430-441, 1975.

14. Gonzalez-Duarte A, Saniger-Alba Mdel M and Higuera-Calleja J: Cryptococcal meningitis in HIV-negative patients with systemic connective tissue diseases. Neurol Res 37: 283-287, 2015.

15. Louro R, Ferreira R, Pinheiro C, Parada H, Faria D and Monteiro E: Fungal meningitis in an immunocompetent patient. Clin Drug Investig 33 (Suppl 1): S47-S50, 2013.

16. Portelinha J, Passarinho MP, Almeida AC and Costa JM: Bilateral optic neuropathy associated with cryptococcal meningitis in an immunocompetent patient. BMJ Case Rep 2014: pii, 2014.

17. Saigal G, Post MJ,Lolayekar S and Murtaza A: Unusual presentation of central nervous system cryptococcal infection in an immunocompetent patient. AJNR. Am J Neuroradiol 26: 2522-2526, 2005.

18. McMullan BJ, Sorrell TC and Chen SC: Cryptococcus gattii infections: Contemporary aspects of epidemiology, clinical manifestations and management of infection. Future Microbiol 8: 1613-1631, 2013. 
19. Espino Barros Palau A, Morgan ML, Foroozan R and Lee AG: Neuro-ophthalmic presentations and treatment of Cryptococcal meningitis-related increased intracranial pressure. Can J Ophthalmol 49: 473-477, 2014.

20. Khairullah S, Sulaiman H, Yahya F, Jasmin R, Cheah TE, Sockalingam S, Bick J and Chin Teck NG: Cryptococcal meningitis and SLE: A diagnostic and therapeutic challenge. Acta Reumatol Port 39: 254-258, 2014

21. Patil SA, Katyayani S and Arvind N: Significance of antibody detection in the diagnosis of cryptococcal meningitis. J Immunoassay Immunochem 33: 140-148, 2012.
22. Sarkis RA, Mays M, Isada C and Ahmed M: MRI findings in cryptococcal meningitis of the non-HIV population. Neurologist 19: 40-45, 2015.

23. Day JN, Chau TT, Wolbers M, Mai PP, Dung NT, Mai NH, Phu NH, Nghia HD, Phong ND, Thai CQ, et al: Combination antifungal therapy for cryptococcal meningitis. N Engl J Med 368: 1291-1302, 2013.

24. Lui G, Lee N, Ip M, Choi KW, Tso YK, Lam E, Chau S, Lai R and Cockram CS: Cryptococcosis in apparently immunocompetent patients. QJM 99: 143-151, 2006. 